\title{
Automated Quadrat Analysis Technique for Particle Dispersion Quantification in Oxide Dispersion Strengthened Alloys
}

\author{
Anna C. Weiss ${ }^{1}$, Txai T. Sibley ${ }^{1}$, Bryan A. Webler ${ }^{1}$ and Yoosuf N. Picard ${ }^{1}$ \\ ${ }^{1 .}$ Dept. of Materials Science and Engineering, Carnegie Mellon Univ., Pittsburgh PA, USA.
}

Oxide dispersion strengthened (ODS) alloys are composites with a high-density dispersion of oxides throughout a metallic matrix. Oxide dispersion strengthening is important for ultrafine-grained (UFG) metallic materials, which exhibit high strength as a result of submicron grain size. While UFG materials exhibit admirable properties, the high driving force for grain growth lowers their mechanical properties at high temperatures. Oxide dispersion strengthening stabilizes the UFG materials at elevated temperatures by acting as barriers to dislocation motion, limiting grain growth and creep. In this study, the term "dispersion" refers to the spatial distribution of particles in the matrix, where more dispersed particles are more uniform and less dispersed particles are more clustered. Several methods of dispersion quantification exist in the literature, but there is no single well established method for quantifying particle dispersion in ODS alloys. In developing a dispersion quantification method for ODS alloys, it is desirable to have an automated, high-throughput process that is able to quickly analyze numerous micrographs from across an entire bulk sample surface. The quadrat method was chosen for this study for its effectiveness in evaluating clustered dispersions [1], as well as its applicability to automating a high-throughput analysis program.

The quadrat method analyzes dispersion by dividing images into a grid of square "quadrats" and counting the number of particles in each quadrat. In the current study, the image analysis program Fiji, a distribution of ImageJ [2], is used to automate the quadrat method in conjunction with Python scripting. The quadrat program can analyze a series of micrographs that exhibit sufficient contrast between the particles of interest and the matrix (Fig. 1a). Micrographs are recorded in a FEI Quanta 200 and FEI Nova Nanolab SEM under various acceleration voltages and electron detectors. Fiji isolates the particles in each image by applying a thresholding parameter [3] (Fig. 1b). Then, the center of mass of each particle is found, and a quadrat grid is applied (Fig. 1c). The script then counts the number of particles in each quadrat to create a histogram plotting frequency of number of particles per quadrat (Fig. 1d), which can then be fitted to statistical distribution curves in order to quantify particle dispersion. If the particles are randomly dispersed, the histogram will fit closer to a Poisson distribution, while a more clustered dispersion will fit closer to a negative binomial distribution. Additionally, a skewness parameter can be calculated from the mean and standard deviation of number of particles per quadrat, which indicates the degree of clustering. This skewness value is useful for comparing the degree of dispersion between similar samples of different processing conditions.

This automated approach is applied to a series of $\mathrm{Fe}-\mathrm{Y}$ alloys processed under a variety of processing conditions designed to create nanoscale $\mathrm{Y}_{2} \mathrm{O}_{3}$ oxide particles by internal oxidation of $\mathrm{Fe}_{17} \mathrm{Y}_{2}$ intermetallic, followed by equal-channel angular pressing (ECAP) in order to refine and distribute the $\mathrm{Y}_{2} \mathrm{O}_{3}$ particles in the Fe matrix. Particle dispersion is correlated to oxidation conditions and number of ECAP cycles. Furthermore, particle dispersion is compared to subsequent Fe grain growth stabilization by comparing the Fe matrix microstructure of oxidized Fe-Y alloys before and after annealing. Future work will include adapting this automated quadrat analysis program into a plugin for ImageJ, allowing other users to quickly quantify particle dispersion in ODS alloys using the quadrat method [4]. 
References:

[1] PA Karnezis, G Durrant and B Cantor, Materials Characterization 40 (1998), p. 97-109.

[2] J Schindelin et al, Nature Methods 9 (2012), p. 676-682.

[3] T Ridler and S Calvard, IEEE Transactions on Systems, Man, and Cybernetics 8 (1978), p. 630-632.

[4] Research supported by NSF CMMI Award No. 1436064. The authors acknowledge use of the Materials Characterization Facility at Carnegie Mellon University supported by grant MCF-677785.
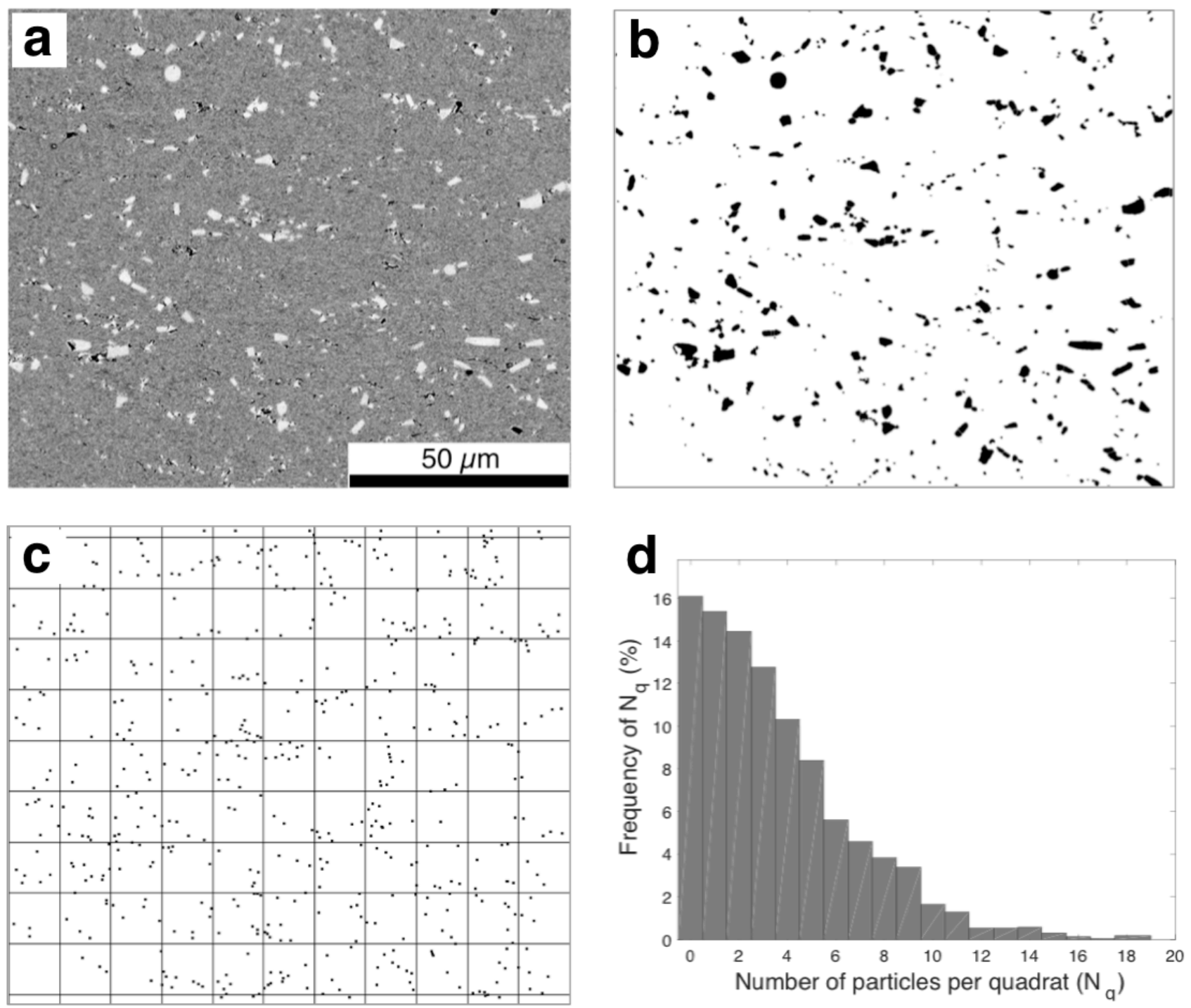

Figure 1. Quadrat analysis steps: a) initial SEM micrograph of an ODS alloy where bright features denote $\mathrm{Fe}_{17} \mathrm{Y}_{2}$ particles in an Fe matrix, b) the isolated particles thresholded in Fiji, c) quadrat grid over the center of mass coordinates, and d) a frequency histogram plotted from the sample represented by Figure (a). 